The Astrophysical JouRnal, 505:363-368, 1998 September 20

(C) 1998. The American Astronomical Society. All rights reserved. Printed in U.S.A.

\title{
MAGNETIC ANNIHILATION AND RECONNECTION IN TWO DIMENSIONS
}

\author{
P. G. Watson AND I. J. D. Craig \\ Department of Mathematics, University of Waikato, Hamilton, New Zealand; pgwatson, i.craig@math.waikato.ac.nz \\ Received 1997 May 13; accepted 1998 April 29
}

\begin{abstract}
The problems of incompressible, planar, magnetic annihilation and reconnection are discussed. We first emphasize that steady-state reconnection solutions can be constructed from annihilation models involving harmonic velocity fields. We show, however, that the only harmonic velocity profile capable of supporting inviscid magnetic annihilation is the traditional stagnation point flow $\phi=-\alpha x y$. The implication is that further steady-state planar reconnection models derived from annihilation solutions are impossible. We go on to show that certain classes of nonharmonic stream functions allow reconnection solutions to be developed, once the constraint of time independence is relaxed. In particular, we construct an exact reconnection model based on cellular inflows into a periodic assemblage of magnetic X-points.
\end{abstract}

Subject headings: MHD - plasmas

\section{INTRODUCTION}

Although magnetic reconnection theory in three dimensions is not well developed, the view is often advanced that two-dimensional planar reconnection is quite well understood. This optimism is often supported by schematic magnetic merging diagrams that illustrate how magnetic reconnection is expected to occur in rather idealized geometries. There is no doubt that such models provide useful heuristics, say, in the interpretation of data, but a number of controversial problems remain (Biskamp 1994). These problems can be expected to recur in more complicated threedimensional reconnection models.

What are the main problems associated with traditional reconnection models based on incompressible merging at symmetric four-lobe neutral points? In the first place, the "magnetic slingshot" that ejects plasma from the sheet can only be very weak. This follows from the fact that the separatrices of the field cannot meet in a traditional $\mathrm{X}$-point; rather, they osculate to form a quasi-onedimensional sheet in the vicinity of the null (Priest \& Cowley 1975; Biskamp 1994).

Another difficulty resides in the amplitude of the flow field. If the flow field is sub-Alfvénic, then plasma is unable to advect across the magnetic separatrices (Craig \& Rickard 1994). This means that "reconnection" in the conventional sense cannot occur; the best that can be achieved - given that the current density has to remain constant on each field line-is stationary fronts of enhanced diffusion extending from the neutral point to the outer boundaries of the field. The ohmic dissipation rate can still be fast (Craig \& Henton 1994), but the transfer of flux between adjacent lobes of the $\mathrm{X}$-point is overruled.

More generally, Priest et al. (1994) have formalized the problem of steady, sub-Alfvénic merging in terms of an "antireconnection theorem" valid for plasmas of arbitrary compressibility. They point out that reconnection can occur only if account is taken of finite fluid viscosity or nonlinearity. We note that exact magnetic reconnection solutions that include the effect of viscosity are available in both two and three dimensions, and their reconnection rates are found to be essentially independent of the fluid viscosity level (Fabling \& Craig 1996). However, these solutions are reconnective, not because they include viscosity, but because they break the flow/field symmetries invoked in the standard models (Craig \& Fabling 1996).

In this paper we try to construct reconnection solutions using the superposition methods of Craig \& Henton (1995) and Watson \& Craig (1997a, 1997b). Following Watson, Priest, \& Craig (1998), we term models generated in this manner "reconnective diffusion" solutions, because magnetic flux is transported across some separatrices by mass transfer and across others by ohmic diffusion. The steadystate MHD equations are introduced in $\S 2$. The basis of our approach, as discussed in $\S 3$, is to derive reconnection models by breaking the symmetry of simple annihilation solutions. In $\S 4$ we focus on harmonic flow solutions, because they are the only type of flows that can support steady-state reconnective diffusion solutions based on the superposition method. We show, using an antireconnection theorem, that simple harmonic flows cannot directly support reconnective solutions (i.e., before superposition). More surprisingly, we demonstrate that general harmonic flows do not even support simple annihilation solutions. It follows that the superposition method is inapplicable except in the case of the stagnation point flow $v=\alpha(-x, y)$ (Craig $\&$ Henton 1995). In $\S 5$ we point out that nonharmonic flows can be used to develop reconnective diffusion solutions provided the assumption of steady-state merging is relaxed. In particular, we develop a dynamic reconnection solution based on the periodic inflow model $v=\alpha[-\sin$ $(k x), k \cos (k x) y]$.

\section{MHD EQUATIONS}

The equations that govern the behavior of a steady-state, magnetized plasma with velocity $\boldsymbol{v}$, magnetic field $\boldsymbol{B}$, density $\rho$, electric field $\boldsymbol{E}$, and gas pressure $p$ can be written in the following nondimensional form:

$$
\begin{gathered}
\rho(\boldsymbol{v} \cdot \nabla) v=-\nabla p+(\nabla \times B) \times B, \\
E+v \times B=\eta \boldsymbol{J}, \\
\nabla \cdot \boldsymbol{B}=0, \\
\nabla \cdot(\rho v)=0,
\end{gathered}
$$

where $\eta$ is the dimensionless resistivity, and $\boldsymbol{J}=\boldsymbol{\nabla} \times \boldsymbol{B}$ is the current density. The resistivity, $\eta$, need not be assumed constant and may be taken as a function of the state of the 
plasma; in particular, we might consider plasmas where the resistivity is enhanced by current driven instabilities. The resistivity is, however, very small: its magnitude is the inverse Lundquist number, of order $10^{-12}$ for the solar corona.

If the system we are considering is two-dimensional, then the magnetic field $\boldsymbol{B}$ may be expressed in the form $\boldsymbol{B}=\boldsymbol{\nabla} \times\{\psi(x, y) \hat{z}\}$. The gas pressure can then be removed from equations (1)-(4), by taking the curl of the momentum equation, to give

$$
\begin{gathered}
\nabla \times\{\rho(\boldsymbol{v} \cdot \nabla) \boldsymbol{v}\}=-\left[\nabla^{2} \psi, \psi\right] \hat{z}, \\
\boldsymbol{v} \cdot \nabla \psi=E+\eta \nabla^{2} \psi, \\
\nabla \cdot(\rho v)=0,
\end{gathered}
$$

where $E$ is the $z$-component of a constant electric field $\boldsymbol{E}=$ $E \hat{z}$, and $[f, g]=f_{x} g_{y}-f_{y} g_{x}$ (here subscripts denote partial differentiation).

If we consider a constant-density, incompressible plasma, then we may make further simplifications by setting the plasma density $\rho=1$ and writing $v=\nabla \times\{\phi(x, y) \hat{z}\}$. The governing equations, written in terms of the new variables $\phi$ and $\psi$, now reduce to

$$
\begin{gathered}
{\left[\nabla^{2} \phi, \phi\right]=\left[\nabla^{2} \psi, \psi\right],} \\
{[\psi, \phi]=E+\eta \nabla^{2} \psi .}
\end{gathered}
$$

The relative simplicity of this system makes it possible to construct exact models for magnetic annihilation and reconnection.

\section{EXACT ANNIHILATION AND RECONNECTION SOLUTIONS}

The annihilation of antiparallel magnetic fields in incompressible plasmas (e.g., Sonnerup \& Priest 1975) can be described by equations (8) and (9) by assuming that the flux function $\psi(x, y)$ is a function of only one variable, i.e., $\psi=\psi(x)$ (Craig \& Henton 1995). Substituting this form for $\psi$ into equations (8) and (9) shows that $\left[\nabla^{2} \phi, \phi\right]=0$ and $\phi_{y}$ is a function of $x$ only. These constraints admit three pos- sible forms for the stream function, namely,

$$
\phi=-\alpha x y, \quad-\alpha \sin (k x) y, \quad \alpha \sinh (k x) y
$$

The three resulting solutions for $\psi$ all represent the steadystate annihilation of magnetic field in the vicinity of a flow stagnation point located at the origin.

Craig \& Henton (1995) went on to show that in certain cases annihilation solutions may be modified via a suitable superposition of $\phi$ and $\psi$ to give reconnection solutions. For the superposition to yield exact solutions, we require $\phi$ to be a harmonic function, i.e., $\nabla^{2} \phi=0$. This implies $\phi=-\alpha x y$ is the only one of the three annihilation solutions outlined above that can be converted to a reconnective diffusion solution in this manner. The other flow profiles lead to approximate "quasi-steady" solutions using the methods of Watson \& Craig (1997a). However, if the constraint of time independence is relaxed (as discussed in $\S 5)$ these flow profiles can be used to construct dynamic reconnective diffusion solutions.

The exact steady-state solution is given by

$$
\begin{aligned}
& \phi^{*}=\phi+\lambda \psi=-\alpha x y-\frac{\lambda E x^{2}}{2 \eta}{ }_{1} F_{2}\left(1, \frac{3}{2}, 2,-\mu^{2} x^{2}\right), \\
& \psi^{*}=\lambda \phi+\psi=-\lambda \alpha x y-\frac{E x^{2}}{2 \eta}{ }_{1} F_{2}\left(1, \frac{3}{2}, 2,-\mu^{2} x^{2}\right),
\end{aligned}
$$

where $\phi$ and $\psi$ are the annihilation solution, $\phi^{*}$ and $\psi^{*}$ are the reconnection solution, ${ }_{1} F_{2}(a, b, c, z)$ is a generalized hypergeometric function (Spanier \& Oldham 1987), and $\mu^{2}=\left(1-\lambda^{2}\right) \alpha / 2 \eta$.

The transition from annihilation to reconnective diffusion solution is summarized in Figure 1. It is clear that breaking the flow symmetry of the annihilation solution allows curved field lines to reconnect across the neutral point. Note that the momentum brackets are nonvanishing in the reconnection solution, i.e., $\left[\nabla^{2} \phi^{*}, \phi^{*}\right]=\left[\nabla^{2} \psi^{*}, \psi^{*}\right]$ $\neq 0$. In fact, according to the theorem discussed below,
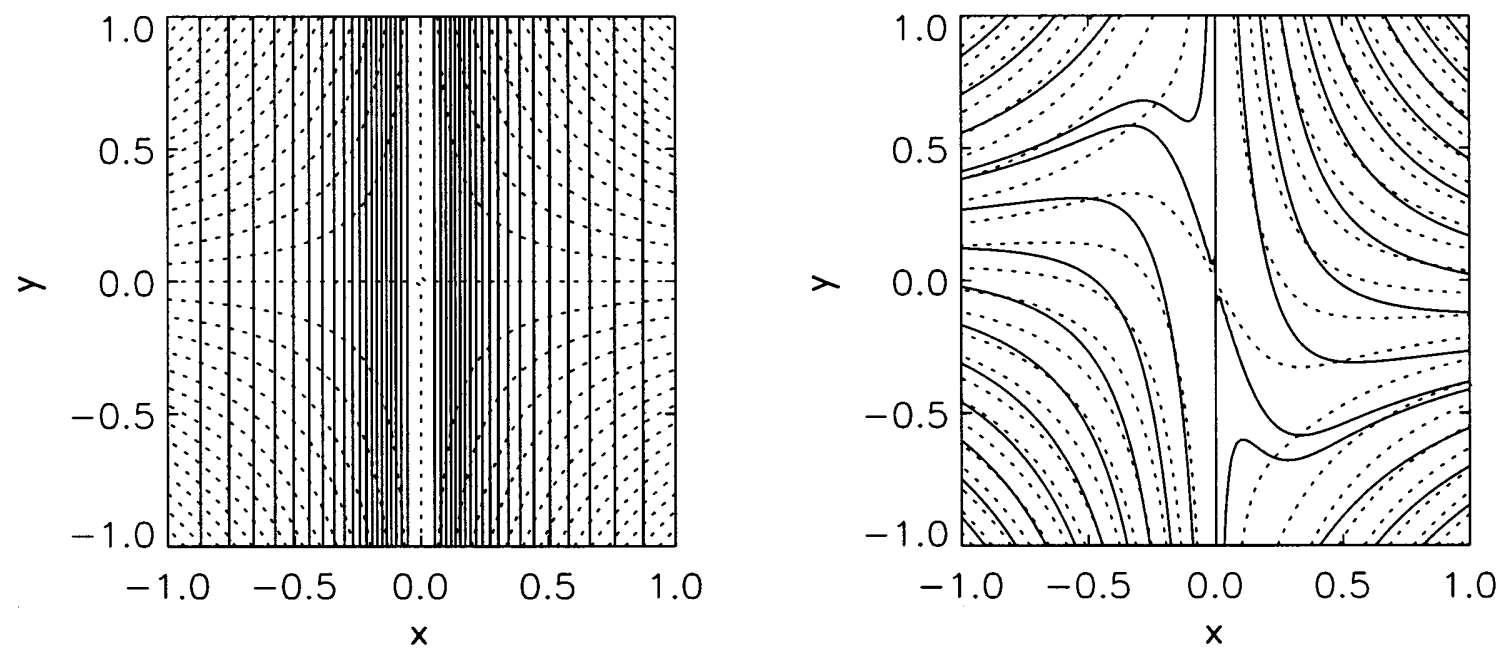

FIG. 1.-Diagram comparing the planar annihilation (left) and reconnection (right) solutions (dotted lines, streamlines; solid lines, magnetic field-lines). Note that in the superposed reconnection solution the symmetry about the $x$-axis is broken. Parameters are $\alpha=1, \eta=0.01$, and $E=0.05$ for the annihilation model, with $\lambda=0.75$ for the reconnection model. 
reconnection is impossible if the momentum brackets vanish identically.

\section{HARMONIC FLOW SOLUTIONS}

\subsection{Introduction}

The previous discussion shows that steady-state reconnective diffusion solutions can be developed from annihilation solutions by an appropriate superposition of the velocity and magnetic fields. For the construction to be exact, we require that the harmonic condition $\nabla^{2} \phi=0$ is satisfied. Before attempting to find annihilation solutions, let us first establish that harmonic flow models cannot directly support reconnection solutions.

\subsection{The Antireconnection Theorem}

Using equations (5) and (6), it is possible to determine certain conditions under which reconnection cannot occur. Antireconnection results of this type have already been developed for sub-Alfvénic flows by Craig \& Rickard (1994) and Priest et al. (1994). The form developed below generalizes previous results by taking account of finite amplitude flows. The central requirement is that $\left[\nabla^{2} \psi, \psi\right]=0$, so that the flow does not necessarily have to be sub-Alfvénic; it just has to satisfy $\nabla \times\{\rho(v \cdot \nabla) v\}=0$. The crucial point is that if $\left[\nabla^{2} \psi, \psi\right]=0$ and $J=-\nabla^{2} \psi$ is continuous, then we require $J$ to be a function of $\psi$; i.e., the current density must be constant on a field line.

THEOREM: Steady two-dimensional reconnection is impossible for plasmas in which the bracket $\left[\nabla^{2} \psi, \psi\right]$ vanishes, at least provided the plasma contains only isolated null points of the field and the resistivity is only a function of the current density, $\eta=\eta(J)$.

Proof. The bracket $\left[\nabla^{2} \psi, \psi\right]$ can vanish identically only if $\nabla^{2} \psi=-J(\psi)$. In this case the induction equation (2) may be rewritten in the form

$$
\boldsymbol{v} \times \boldsymbol{B}=[\bar{\eta}(\psi) J(\psi)-E] \hat{z}=f(\psi) \hat{z},
$$

where $\bar{\eta}(\psi)=\eta[J(\psi)]$.

Let $\psi=0$ label the separatrices of the field, as defined by the totality of field lines threading the null point. Since $\boldsymbol{v} \times \boldsymbol{B}$ vanishes at the null, we must have $f(0)=0$. However, since $f(\psi)$ must remain constant along all field lines, $\boldsymbol{v} \times \boldsymbol{B}$ must also vanish along the lines $\psi=0$. If we consider fields with only isolated null points, then at an arbitrary point along a separatrix $\boldsymbol{B} \neq 0$. As $\boldsymbol{v} \times \boldsymbol{B}$ must vanish, our only choices are that $\boldsymbol{v}$ is parallel to $\boldsymbol{B}$ or $\boldsymbol{v}=0$. In either case it is clear that there is no motion across the separatrix and that reconnection is impossible. Q.E.D.

Note that the argument fails if $\boldsymbol{B}$ vanishes along a line (which by definition must also be a field line), as the condition $v \times B=0$ no longer places any restrictions on $v$. A second point is that the antireconnection theorem is valid independent of the compressibility of the plasma, at least provided $\left[\nabla^{2} \psi, \psi\right]=0$.

As a simple application, we note that incompressible reconnection driven by a harmonic velocity field cannot occur, for in this case the antireconnection theorem can be rigorously applied:

$$
\nabla^{2} \phi=0 \Rightarrow\left[\nabla^{2} \phi, \phi\right]=\left[\nabla^{2} \psi, \psi\right]=0 .
$$

Indeed, any finite amplitude incompressible flow for which $\nabla^{2} \phi=-\omega(\phi)$, where $\omega(\phi)$ can be any function of $\phi$, must obey the antireconnection theorem. By contrast, in sub-Alfvénic applications (Craig \& Henton 1994; Priest et al. 1994) the vanishing of the momentum brackets is only approximately achieved.

One consequence of these results is the impossibility of constructing reconnection solutions for the simple stagnation point flow model $\phi=-\alpha x y$. This result is apparently nontrivial: witness the kinematic reconnection models of Parker (1973) based on stagnation point flow! However, if we recall that a pure annihilation solution is possible in which $\psi=\psi(x)$, we can still develop a reconnection model using the superposition argument of Watson \& Craig (1997a). This superposition breaks the symmetry of the stagnation point flow field and leads directly to the Craig \& Henton (1995) solution (11), discussed in $\S 3$.

\subsection{A Scheme for Constructing Reconnection Solutions}

Although the antireconnection theorem overrules reconnection models based on harmonic flow profiles, the possibility of constructing annihilation solutions remains open. Suppose we take some harmonic flow field using the construction $\phi=\operatorname{Re}\left(a_{n} z^{n}\right)$, where $z=x+i y$ and $a_{n}$ is a complex normalization constant, and use this to develop an annihilation solution $\psi$. We can now invoke the superposition $\phi^{*}=\phi+\lambda \psi, \psi^{*}=\psi+\lambda \phi$ to develop a full reconnective diffusion solution (Watson \& Craig 1997a). We know this construction leads to an exact reconnection solution for the $n=2$ flow field, $\phi=-\alpha x y$, so we might expect success for the higher order harmonic flow fields. The arguments given below, however, show that a consistent annihilation model can only be constructed in the case of the traditional stagnation point flow.

\subsection{Can Harmonic Flows Support Magnetic Annihilation?}

It is well known that the advection of a fluid line element provides an exact description of the magnetic field evolution in the absence of resistive effects. For an incompressible plasma, the identification of $\boldsymbol{B}$ with a fluid line element is formalized by the Cauchy solution (Craig \& Sneyd 1990). Thus a flow field that stretches the line element has the effect of increasing the amplitude of the advected magnetic field. The stretching flows of the various stagnation point models are thus associated with dramatic flux pile-up in the vicinity of the outflow separatrices.

Figure 2 shows the hypothetical accumulation of the field about the outflow separatrices in the case of $n=2, n=3$, and $n=4$ harmonic solutions. We are imagining here-as the symmetry demands - that the effects of the plasma resistivity are localized to narrow strips of intense current overlying the outflow separatrices. As indicated in the diagrams, the development of the current layer can be anticipated by the stretching associated with a localized blob of plasma advected by the inflow.

The problem is that while the $n=2$ annihilation "model" drawn in Figure $2 a$ is physically realizable, the higher $n$ "models" have an inherent contradiction. Consider the $n=4$ model shown in Figure $2 c$. From the antireconnection theorem we know that the current must have the constant value $J=E / \bar{\eta}(0)$ along the separatrices. On the $y=0$ separatrix, the contribution to $J=-\nabla^{2} \psi$ must come entirely from $\psi_{y y}$, because $\psi$ is constant in the $x$-direction (along the separatrix). However, the converse must be true along the $x=0$ separatrix; i.e., the only contribution to $J$ must come from $\psi_{x x}$. It follows that $\psi$ must contain 

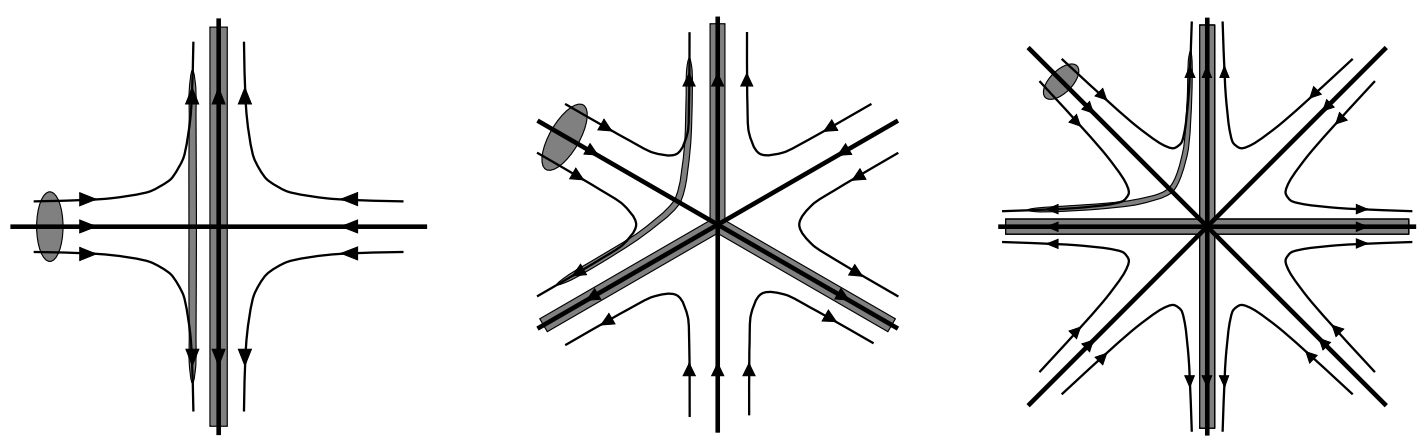

FIG. 2.-Schematic diagram showing the accumulation of magnetic field in the vicinity of outflow separatrices in the case of $n=2(l e f t), n=3($ middle), and $n=4$ (right) harmonic flows. In an ideal plasma, a blob of fluid and the magnetic field embedded within it are advected toward the outflow separatrices and continually stretched. This stretching of the plasma leads to the build-up of the magnetic field. If resistivity is included in the model, the continual build-up of the field eventually saturates, leading to the formation of resistive current layers aligned with the outflow separatrices.

unphysical discontinuities close to the origin unless $E=0$. But if $E=0$ we obtain the physically uninteresting solution, $\psi=k \phi$, corresponding to flow along the field lines. Even more simply, at least for $n=4$, the vanishing of $E$ follows by taking a 5 point finite difference approximation for $\nabla^{2} \psi$ at the origin. Since all grid points lie on the separatrices $\psi=0$, $\nabla^{2} \psi=0$ trivially vanishes.

A similar argument, invoking local coordinates tangent and normal to the separatrices, may be used to overrule viable $E \neq 0$ annihilation solutions for any harmonic flow field in which multiple outflow separatrices intersect at a nonzero angle. In fact, the argument only fails for the $n=2$ case, which possesses a single strip of current. Therefore we must abandon all notions of developing annihilation solutions for higher order harmonic flow fields.

In summary, the absence of annihilation solutions for $n>2$ means that the superposition method cannot be used to generate any two-dimensional steady-state reconnective diffusion models beyond that of Craig \& Henton (1995). While more general three-dimensional superposed solutions are still possible (see Watson \& Craig 1997a, 1997b), the physical implication of our present results is that flow profiles involving strong symmetries cannot support pure magnetic annihilation models.

\section{DYNAMIC RECONNECTION SOLUTIONS}

\subsection{Introduction}

We now demonstrate that annihilation solutions based on nonharmonic flows can be used to develop reconnection solutions. To do this we must generalize to time-dependent reconnection.

Suppose, for definiteness, we invoke the periodic annihilation flow field $\phi=-\alpha \sin (k x) y$ of equation (10) as a basis for a dynamic reconnection model. Any reconnection solution must satisfy the time-dependent system

$$
\begin{gathered}
\frac{\partial \omega}{\partial t}+[\omega, \phi]=[J, \psi], \\
\frac{\partial \psi}{\partial t}+[\psi, \phi]=\eta \nabla^{2} \psi,
\end{gathered}
$$

where $J=-\nabla^{2} \psi$, and we have introduced the fluid vorticity $\omega=-\nabla^{2} \phi$. If we take the forms

$$
\phi=-\alpha \sin (k x) y+f(x, t),
$$

$$
\psi=-\lambda(t) \alpha \sin (k x) y+g(x, t)
$$

we find that the induction equation implies

$$
\lambda(t)=\lambda_{0} \exp \left(-\eta k^{2} t\right),
$$

where $\lambda_{0}$ is a constant. The momentum and induction equations now govern the evolution of the nonlinear disturbance fields $f$ and $g$ via

$$
\begin{gathered}
f_{x t}=\alpha \sin (k x)\left[f_{x x}-\lambda g_{x x}\right]-\alpha k \cos (k x)\left[f_{x}-\lambda g_{x}\right], \\
g_{t}=\alpha \sin (k x)\left[g_{x}-\lambda f_{x}\right]+\eta g_{x x} .
\end{gathered}
$$

These equations generally require a numerical solution. A special case occurs when $\lambda=0$. This corresponds to a timedependent annihilation solution in which $f$ can be taken to vanish identically. Steady-state solutions based on flows of this form are closely related to the cellular flow patterns discussed by Jardine et al. (1992); however, their steadystate model includes viscosity, which we have not included in our treatment of the problem.

\subsection{A Dynamic Solution for an Assemblage of $X$-Points}

Let us summarize the main properties of the reconnection solution based on equations (16) and (17) above. We shall not go into detail, since the solution can be understood in much the same way as the dynamic, harmonic flow solutions of Craig \& McClymont (1997) and the fully threedimensional model of Craig \& Fabling (1998). The central requirement for fast reconnection is that the background flow is strong enough to localize the magnetic field disturbance and drive flux pile-up sheets. This requires $|\lambda|<1$. The new feature of the present model is the cellular reconnection structure. This involves current sheets supported by plasma inflows into alternate stagnation points of the flow.

Suppose, for example, that we take some longwavelength, periodic initial disturbance for the magnetic field, say $g(x, 0)=g_{0}(x)$. Provided that $|\lambda|<1$, the disturbance will localize about the stagnation points of the flow associated with inflow in the $x$-direction (i.e., at alternate stagnation points). This localization occurs over a timescale governed by the strength $\alpha$ of the background flow field. The disturbance can then drive strong current sheets, leading to rapid ohmic diffusion.

Figure 3 shows a typical solution at the time of maximum current in the sheet. By this time the current layer has collapsed to the width $\Delta x \propto \eta^{1 / 2}$ and the current density has achieved the level $J \propto \eta^{-1}$. These scalings are consistent 

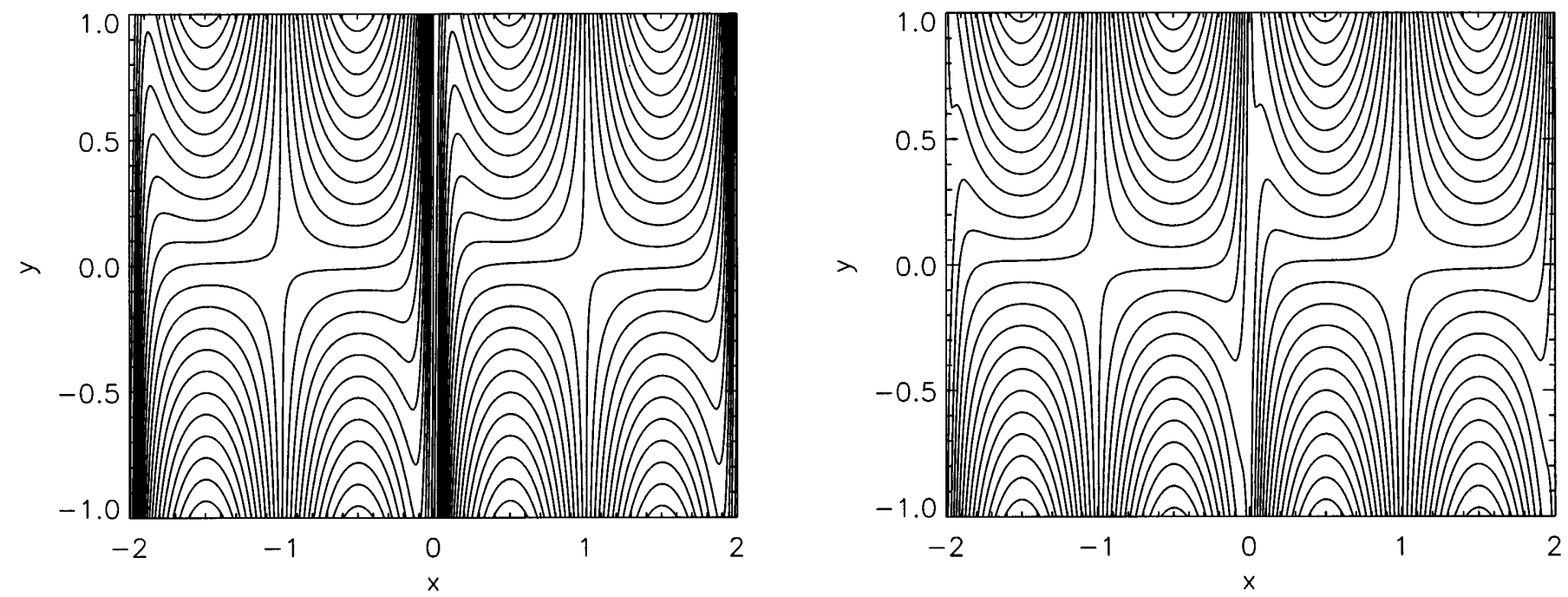

FIG. 3.-Streamlines and field lines for a dynamic reconnection solution at the time of maximum current. There is a periodic assemblage of $X$-points, but current sheets form only at neutral points associated with material inflow. Parameters are $\alpha=1, \eta=0.003, \lambda_{0}=0.5$, and $k=\pi$, with $g_{0}(x)=\sin (\pi x)$.

with fast ohmic dissipation (Craig \& Henton 1995; Craig \& Fabling 1996).

Indeed, we can go a long way toward understanding this solution by comparing its scalings with those calculated by Craig \& Fabling (1998) for dynamic reconnection at an isolated three-dimensional X-point. Craig \& Fabling's model can be made two-dimensional by setting their parameter $\kappa$ equal to unity. We can then compare numerically generated scalings for the present model: see Figures $4 a$ and $4 b$, with their derived scalings for the current maximum and the time to current maximum

$$
J_{\max } \propto \eta^{-1}, \quad T_{\max } \simeq \frac{1}{2 \alpha^{-}} \ln \left(\frac{\alpha^{-}}{\eta}\right), \quad \alpha^{-} \simeq \alpha k\left(1-\lambda_{0}^{2}\right) .
$$

Although their theory cannot strictly be applied to sinusoidal flow profiles, we expect the underlying arguments to remain valid. In fact, the scalings obtained by Craig \& Fabling (1998) should remain valid for any flow profile that approximates stagnation point flow in the vicinity of the neutral point. This is certainly true of the present sinusoidal flow model, at least provided $k$ remains $O(1)$.

There is, however, a caveat to the interpretation of these results. Although, for $|\lambda|<1$, the resistive release is formally fast-independent of any positive power of the resistivity - very large "external" hydromagnetic pressures are required to sustain the merging. This is a recurring difficulty in all flux pile-up annihilation and reconnection solutions: it means that flux pile-up cannot be sustained indefinitely with reductions in the plasma resistivity. In practice, the amplitude of the disturbance must be limited to maintain realistic hydromagnetic pressures. Without going into the details of the flux saturation amplitude, we note that the problem is considerably eased when the solution is extrapolated to three dimensions (see Craig, Fabling, \& Watson 1997).

Finally, we mention that, although we have only demonstrated one time-dependent reconnection solution, there exist more general planar solutions based on fully twodimensional current structures. We are currently investigating these solutions. In fact, in a subsequent paper we will
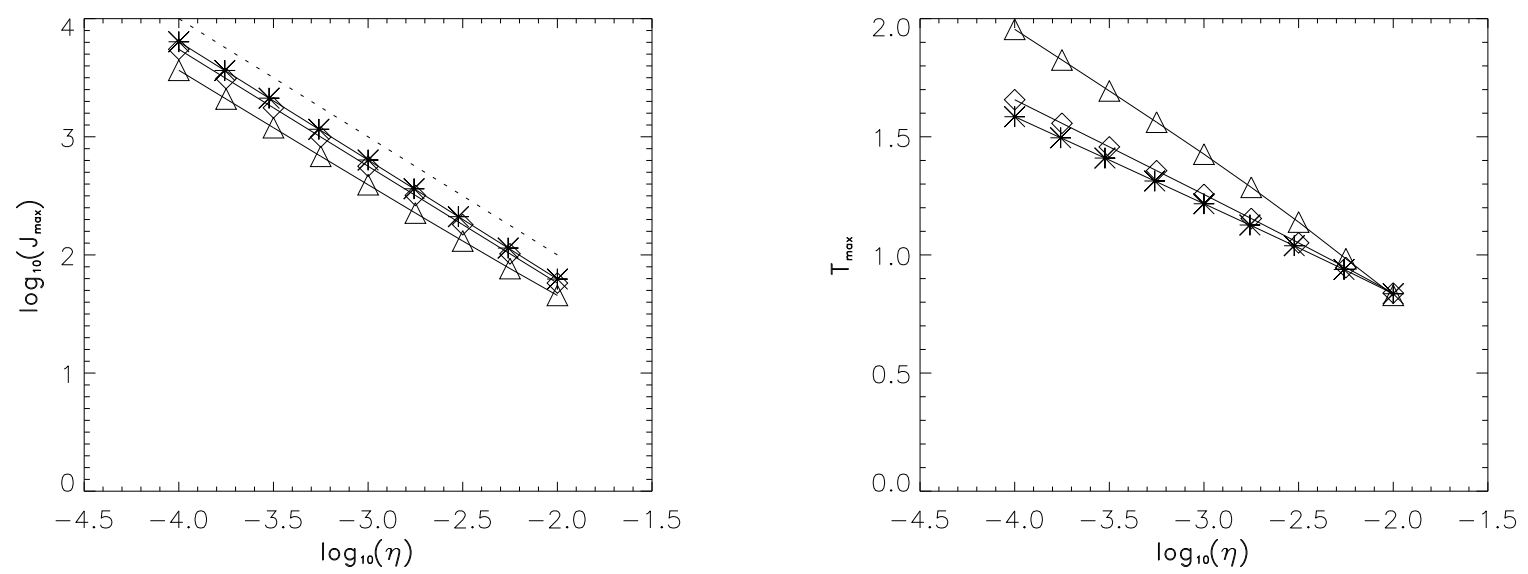

FIG. 4. - Left: Plot of $\log _{10}\left(J_{\max }\right)$ vs. $\log _{10}(\eta)$ for three simulations of eqs. (16)-(17), with $\alpha=1, k=\pi, \lambda_{0}=0$ (asterisks), $\lambda_{0}=0.25$ (diamonds), and $\lambda_{0}=0.5$ (triangles) and the initial condition $g_{0}(x)=\sin (\pi x)$. The slope of the dotted line represents the theoretical $1 / \eta$ scaling. Right: Plot of $T_{\max }$ (the time taken to reach current maximum) vs. $\log _{10}(\eta)$ for the same runs as in the sleft panel. The agreement with the theory of Craig \& Fabling (1998) is surprisingly good. 
show that more general solutions can be constructed that include both the effects of time dependence and viscosity.

\section{CONCLUSIONS}

We have shown that it is difficult to construct analytical steady-state, planar reconnection solutions by invoking highly symmetric flow topologies. In the first place, the antireconnection theorem shows that harmonic flow fields cannot support any form of steady reconnection. This leaves open the possibility that reconnection solutions can still be developed from annihilation models supported by harmonic flows. We have shown, however, that even annihilation solutions are incompatible with harmonic flows that possess too much symmetry. To quantify "too much," we note that the four-lobe structure of the $n=2$ stagnation point flow solution $\phi=-\alpha x y$ is the most that can be achieved for an exact annihilation solution. This flow symmetry must again be broken if the annihilation model is to be converted into a fully reconnective solution (Craig \& Henton 1995).

We have demonstrated, however, that the range of admissible flow fields can be extended by considering timedependent reconnection models. In particular, global flows of the generic form $\phi=-\alpha e^{i k x} y$ now form a basis for dynamic reconnection solutions (with $\alpha$ and $k$ complex constants). A concrete reconnection example was constructed in $\S 5$. These solutions can model reconnection in a periodic assemblage of $X$-points, such as occurs, for example, in the coalescence instability (Biskamp \& Welter 1980; Rickard \& Craig 1993).

A positive aspect of the present solutions is their natural "extrapolation" into three dimensions. It is well known that the eigenstructure of three-dimensional null points involves "spine" curves and "fan" surfaces (e.g., Priest \& Titov 1996), along which disturbance currents tend to accumulate (Rickard \& Titov 1996). This structure makes it difficult to extrapolate any planar model that maintains a strict parity between the magnetic separatrices-for there is no asymmetry to determine which separatrix plane should collapse to the spine. Notably, in the present reconnection solutions, no parity is maintained between the two separatrix planes: one is associated with the transfer of magnetic flux by advection, the other by resistive diffusion. In fact, it is the current separatrix of the planar model that becomes the fan in the extension to three-dimensional merging (Craig et al. 1997).

The authors wish to thank Richard Fabling for helpful discussions. P. G. W. acknowledges the financial support of a NZ FRST Postdoctoral Fellowship.
Biskamp, D. 1994, Phys. Rep., 237, 181

Biskamp, D., \& Welter, H. 1980, Phys. Rev. Lett., 44, 1069

Craig, I. J. D., \& Fabling, R. B. 1996, AJ, 462, 969 1998, Phys. Plasmas, 5, 635

Craig, I. J. D., Fabling, R. B., \& Watson, P.G. 1997, AJ, 485, 383

Craig, I. J. D., \& Henton, S. M. 1994, AJ, 434, 192 1995, AJ, 450, 280

Craig, I. J. D., \& McClymont, A. N. 1997, AJ, 481, 996

Craig, I. J. D., \& Rickard, G. J. 1994, A\&A, 287, 261

Craig, I. J. D., \& Sneyd, A. D. 1990, AJ, 357, 653

Fabling, R. B., \& Craig, I. J. D. 1996, Phys. Plasmas, 3, 2243

Jardine, M., Allen, H. R., Grundy, R. E., \& Priest, E. R. 1992, J. Geophys.

Res., 97, 4199

Parker, E. N. 1973, J. Plasma Phys., 9, 49

\section{REFERENCES}

Priest, E. R., \& Cowley, S. W. H. 1975, J. Plasma Phys., 14, 271

Priest, E. R., \& Titov, V. S. 1996, Philos. Trans. R. Soc. London, A, 354, 2951

Priest, E. R., Titov, V. S., Vekstein, G. E., \& Rickard, G. J. 1994, J. Geophys. Res., 99, 21467

Rickard, G. J., \& Craig, I. J. D. 1993, Phys. Fluids B, 5, 956

Rickard, G. J., \& Titov, V.S. 1996, AJ, 472, 840

Sonnerup, B. U. Ö., \& Priest, E. R. 1975, J. Plasma Phys., 14, 283

Spanier, J., \& Oldham, K. B. 1987, An Atlas of Functions (New York: Hemisphere)

Watson, P. G., \& Craig, I. J. D. 1997a, Phys. Plasmas, 4, 101

. 1997b, Phys. Plasmas, 4, 110

Watson, P. G., Priest, E. R., \& Craig, I. J. D. 1998, Geophys. Astrophys. Fluid Dyn., in press 